\title{
Neutrino Cross Sections: Models
}

\author{
Marco Martini ${ }^{* \dagger}$ \\ Department of Physics and Astronomy, Ghent University, Proeftuinstraat 86, B-9000 Gent, \\ Belgium \\ E-mail: martini.marco@gmail.com
}

In the present and future accelerator-based neutrino oscillation experiments nuclear targets (such as $\mathrm{C}, \mathrm{O}, \mathrm{Ar}$ and $\mathrm{Fe}$ ) are involved. Hence the knowledge of neutrino-nucleus scattering is very important. In particular it is crucial for the determination of the neutrino energy which enters the expression of the oscillation probability, since this energy is reconstructed from the final states of the neutrino-nucleus reaction. The status of the different theoretical approaches treating the open channels in the few-GeV region, i.e. the quasielastic, the pion production and the multinucleon emission, is reviewed. Special emphasis is devoted to the multinucleon emission channel, which turned to be crucial to explain the unexpected behavior of the charged current quasielastic measurement performed by MiniBooNE. Up to last year, this channel was not included in the generators used for the analyses of the neutrino cross sections and oscillations experiments.

XVI International Workshop on Neutrino Telescopes,

2-6 March 2015

Palazzo Franchetti - Istituto Veneto, Venice, Italy

\footnotetext{
* Speaker.

$\dagger$ I thank Mauro Mezzetto and the organizing committee for the opportunity to present this review and Magda Ericson for a continuous collaboration and for numerous discussions. The financial support of the Interuniversity Attraction Poles Programme initiated by the Belgian Science Policy Office (BriX network P7/12) and of the Research Foundation Flanders (FWO-Flanders) is acknowledged.
} 


\section{Introduction}

Neutrino physics has undergone a spectacular development in the last decade, following the discovery of neutrino oscillations. In the present and future accelerator-based neutrino oscillation experiments nuclear targets, such as ${ }^{12} \mathrm{C},{ }^{16} \mathrm{O}{ }^{40} \mathrm{Ar}$ or ${ }^{56} \mathrm{Fe}$, are involved, hence the knowledge of neutrino-nucleus scattering is very important. In particular it is crucial for the determination of the neutrino energy which enters the expression of the oscillation probability. In accelerator-based experiments the neutrino beams (at difference with respect to electron beams, for example) are not monochromatic but they span a wide range of energies, hence the incoming neutrino energy is reconstructed from the final states of the reaction. This determination is typically done through the charged current quasielastic events, commonly defined as those in which the emission product only includes one charged lepton. The reconstructed energy hypothesis used to obtain the neutrino energy from the measured charged lepton variables (energy and scattering angle) via a two-body formula is that the neutrino interaction in the nuclear target takes place on a nucleon at rest. The identification of the reconstructed neutrino energy with the real one is too crude. Several nuclear effects such as Pauli blocking, Fermi motion, collective aspects of the nuclear response and, very important, multinucleon emission, need to be taken into account.

The fact that in the accelerator-based experiments the incoming neutrino beam exhibits a wide spectrum of energies implies that not only the quasielastic but also other reaction mechanisms, such as for example the pion production, contribute to the neutrino nucleus cross section. The status of the different theoretical approaches treating the open channels in the few-GeV region, i.e. the quasielastic, the pion production and the multinucleon emission, is here reviewed, devoting special emphasis to the multinucleon emission channel.

\section{Quasielastic and multinucleon emission}

The multinucleon emission channel in connection with the quasielastic has attracted a lot of attention in these last years. In fact, as illustrated in Fig. 1, the inclusion of this channel in the quasielastic cross section was suggested $[1,2]$ to be the possible explanation of the MiniBooNE quasielastic total cross section [3], apparently too large with respect to many theoretical predictions employing the standard value of the axial mass. Since the MiniBooNE experiment, as well as others experiments involving Cherenkov detectors, defines a charged current "quasielastic" event as the one in which only a final charged lepton is detected, the ejection of a single nucleon (a genuine quasielastic event) is only one possibility, and one must in addition consider events involving a correlated nucleon pair from which the partner nucleon is also ejected, as discussed first by Marteau et al. in Ref. [4]. This leads to the excitation of 2 particle- 2 hole ( $2 \mathrm{p}-2 \mathrm{~h})$ states; $3 \mathrm{p}-3 \mathrm{~h}$ excitations are also possible. The inclusion in the quasielastic cross section of events in which several nucleons are ejected (np-nh excitations), leads to an excess over the genuine quasielastic value. Martini et al. $[1,2]$ have argued that this is the likely explanation of the MiniBooNE anomaly showing that their evaluation can account for the excess in the cross section without any modification of the axial mass. After this suggestion the interest of the neutrino scattering and oscillation communities on the multinucleon emission channel rapidly increased. Indeed this channel was not included in the generators used for the analyses of the neutrino cross sections and oscillations experiments. Today 


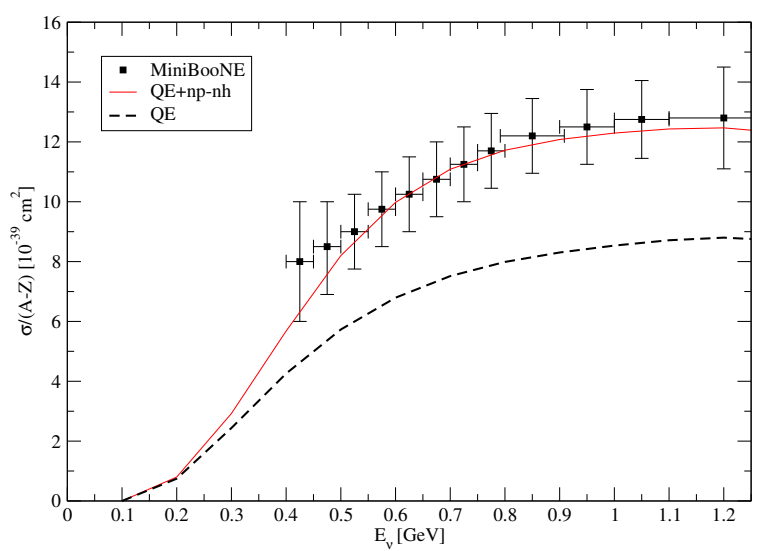

Figure 1: "Quasielastic" cross sections measured by MiniBooNE [3] compared to Martini et al. calculations. The figure is taken from Ref. [1].

there is an effort to include this np-nh channel in several Monte Carlo. Concerning the theoretical situation, nowadays several calculations agree on the crucial role of the multinucleon emission in order to explain the MiniBooNE neutrino [3] and antineutrino [5] data as well as the SciBooNE [6] and T2K [7] inclusive cross sections. Nevertheless there are some differences on the results obtained for this np-nh channel by the different theoretical approaches. The aim of this section is to review the actual theoretical status on this subject.

The theoretical calculations of np-nh excitations contributions to neutrino-nucleus cross sections are actually performed essentially by three groups. There are the works of Martini et al. $[1,2,8,9,10,11,12,13]$, the ones of Nieves et al. [14, 15, 16, 17, 18] and the ones of Amaro et al. $[19,20,21,22,23,24]$. The np-nh channel is taken into account through more phenomenological approaches by Bodek et al. [25] in the so called Transverse Enhancement Model (TEM) and by Lalakulich et al. [26, 27, 28] in GiBUU. In the TEM model an enhanced magnetic form factor, fitted to reproduce the transverse responses in electron scattering, is the effective way to include the multinucleon excitations. In the case of GiBUU the size of the squared matrix element of the neutrino-induced two-nucleon knock-out cross section, is obtained by fitting the neutrino charged current quasielastic MiniBooNE cross section on carbon. In the following we will focus essentially on the results obtained by the three theoretical approaches mentioned above. Considering these three different models, it is important to remind that it exists some differences already at level of genuine quasielastic which can be particularly important when one compares the double differential cross sections. Amaro et al. considered the relativistic superscaling approach (SuSA) based on the superscaling behavior exhibited by electron scattering data. The models of Martini et al. and Nieves et al. are more similar: they start from a local Fermi gas picture of the nucleus. They consider medium polarization and collective effects through the random phase approximation (RPA). Turning to the $2 \mathrm{p}-2 \mathrm{~h}$ sector we remind that there exist several contributions to two-body currents. There are the so called pion-in-flight term, the contact term and the $\Delta$-intermediate state or $\Delta$-MEC term. At level of terminology, some authors refer just to the first two terms as Meson Exchange Currents contributions, like in [1], but actually the most current convention consists into include 
the $\Delta$-term into MEC. In order to preserve the gauge invariance of the theory also the nucleonnucleon $(\mathrm{NN})$ correlation contributions must be taken into account. In the $2 \mathrm{p}-2 \mathrm{~h}$ sector, these three microscopic models are based on the Fermi gas. Even in this simple model an exact relativistic calculation is difficult for several reasons. First of all it involves the computation of 7-dimensional integrals for a huge number of $2 \mathrm{p}-2 \mathrm{~h}$ response Feynman diagrams. Second divergences in the NN correlations sector and in the angular distribution of the ejected nucleons [22, 23] may appear and need to be regularized. Furthermore the calculations should be performed for all the kinematics compatible with the experimental neutrino flux (and not only for some fixed values of the momentum- or energy-transfer, as in the case of the electron scattering where the incoming and outgoing electron energies and momenta are known). For these reasons an exact relativistic calculation is computing very demanding and as a consequence different approximations are employed by the different groups in order to reduce the dimension of the integrals and to regularize the divergences. The choice of subset of diagrams and terms to be calculated presents also important differences. In this connection Amaro et al. consider only the MEC contributions and not the correlations and the correlations-MEC interference terms. Correlations and interference are present both in Martini et al. and Nieves et al. even if Martini et al. consider only the $\Delta$-MEC. On the other hand the treatment of Amaro et al. is fully relativistic as well as the one of Nieves et al. while the results of Martini et al. are related to a non-relativistic reduction of the two-body currents. The interference between direct and exchange diagrams is neglected by Martini et al. and Nieves et al. Another important difference is that Amaro et al. consider the $2 \mathrm{p}-2 \mathrm{~h}$ contribution only in the vector sector while Martini et al. and Nieves et al. also in the axial one. Fully relativistic calculations of Amaro et al. for the axial sector are in progress but not yet included in the published results. Taking into account the existence of all these differences, is not surprising that these models produce different final results. This point is illustrated in Fig. 2 where the MiniBooNE neutrino and antineutrino flux folded double differential CCQE-like cross sections calculated in the different approaches are displayed. For sake of illustartion the results are given for $0.8<\cos \theta_{\mu}<0.9$ as function of the muon kinetik energy. The complete theoretical results in the different bins for neutrinos and antineutrinos are given in Refs. [8, 11] for Martini et al., in Refs. [15, 17] for Nieves et al. and in Refs. [19, 21] for Amaro et al. An updated version of these last results is given by Megias et al. in Ref. [24] from which we take the results reported in the last two panels of Fig. 2. As one can observe from Fig. 2 the results of Martini et al. are in good agreement with the experimental data. In the case of Nieves et al. and Amaro et al. there is a tendency to underestimate the MiniBooNE data. Nevertheless also these results are compatible with MiniBooNE since an additional normalization error of $10 \%$ for neutrino and $17 \%$ for antineutrino is not shown in the error bars. This is the reason why Nieves et al. multiplied the neutrino MiniBooNE data by 0.9 in their figure. An important point is that the relative role of the multinucleon contribution is different for neutrino and antineutrino in the different approaches. The nuclear cross-section difference for neutrinos and antineutrinos stands as a potential obstacle in the interpretation of experiments aimed at the measurement of the CP violation angle, hence has to be fully mastered. In the case of Martini et al. the relative role of the multinucleon contribution is larger for neutrinos, even if it remains important also for antineutrinos, in the case of Nieves et al. it is more or less the same for neutrinos and antineutrinos while for Amaro et al. the relative np-nh contribution is larger for antineutrinos with respect to neutrinos. This difference was even more pronounced in the previous version of the Amaro et al. results [21]. 

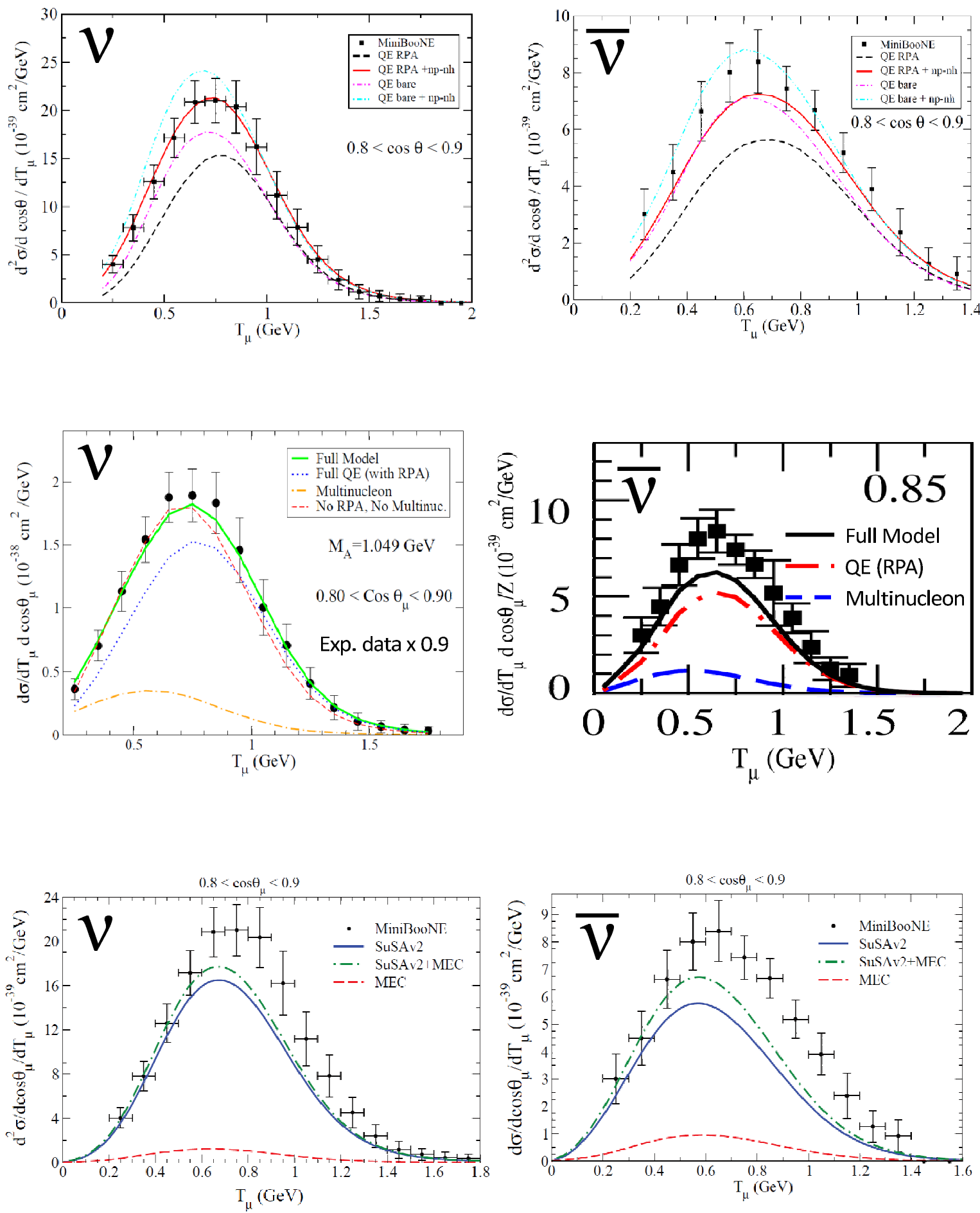

Figure 2: MiniBooNE flux integrated neutrino (left panels) and antineutrino (right panels) CCQE-like double differential cross section on carbon per active nucleon for $0.8<\cos \theta<0.9$ as a function of the muon kinetic energy. Top panels: Martini et al. [8, 11] results. Middle panels: Nieves et al. [15, 17] results. Bottom panels: Megias et al. [24] results representing an update of the Amaro et al. [19, 21] results. 
As discussed in Refs. [2, 21, 13] the difference between the neutrino and antineutrino results is due to the presence in the neutrino-nucleus cross section expression of the vector-axial interference term, which changes sign between neutrino and antineutrino, the basic asymmetry which follows from the weak interaction theory. Due to this vector-axial interference term, the relative weight of the different nuclear responses is different for neutrino and antineutrino. As a consequence also the relative weight of the np-nh contributions is different for neutrino and antineutrino. For example the fact that np-nh contributions are larger for antineutrinos with respect to neutrinos in the case of Amaro et al. is due to the fact that Amaro et al. consider the np-nh contribution only in the vector sector, hence not in the vector-axial interference term. In order to investigate the multinucleon content of the vector-axial interference term, Ericson and Martini have recently considered [13] the difference between the neutrino and antineutrino MiniBooNE quasielastic-like double-differential cross sections. These quantities depend on the neutrino or antineutrino normalized energy flux profiles. In the case of identical ones, the difference provides a direct access to the vector-axial interference term. For the MiniBooNE fluxes Ericson and Martini have tested how much the flux difference influences the combination of the two cross sections, showing that this influence is small. The difference between the MiniBooNE quasielastic-like double-differential neutrino and antineutrino cross sections is rather pure with respect to the vector-axial interference term, which remains dominant. This allows more specific tests of theoretical models on the vector-axial interference term. The model of Martini et al., which includes the np-nh excitations in the vector-axial interference term, gives a good fit for the difference of the MiniBooNE cross sections reproducing well the data in the full range of muon energy and emission angle. This result represents an important test for the presence of the multinucleon component in the vector-axial interference term. A similar conclusion on a relevant two-body current contribution in the vector-axial interference term has been recently obtained by Lovato et al. [29, 30] who calculated the neutral weak current two-body contributions to sum rules and Euclidean responses in ${ }^{12} \mathrm{C}$. The main advantage of the calculation of Lovato et al. is that it is an ab initio microscopic approach which consider a full realistic nuclear interation, fitted on nucleon-nucleon data, with a simultaneous treatment of the two-body current. It can be considered as the state of the art description of nuclear ground state and correlations. The disadvantages are that the currents are non-relativistic, the pion production channel is not included and, most important, these calculations are computing very demanding. Within this approach an evaluation of ${ }^{12} \mathrm{C}$ responses and neutrino cross sections is beyond the present computational capabilities. But the results obtained with this approach offer a benchmark for more phenomenological methods. An example of results that can be obtained within this approach is the already mentioned conclusion of an important two-body current contribution in the vector-axial interference term. Another very interesting result is that two-body current contribution is important also in the axial part of the transverse response. These results support the important $2 \mathrm{p}-2 \mathrm{~h}$ contribution in the axial sector of Martini et al.

Up to now we have discussed the theoretical models in connection with the MiniBooNE cross sections. For the moment the theoretical calculations for the np-nh excitations are restricted to the relatively small energy and momentum transfer, prevalent in the MiniBooNE and T2K experiments. As already mentioned fully relativistic calculations of Amaro et al. for the axial two-body current contributions are still in progress. How the np-nh processes behave at large energy- and momentum transfer is still an open question. Nevertheless Megias et al. [24] applied the model of Amaro et 
al. to neutrino energies of up to $100 \mathrm{GeV}$ and compared their predictions with NOMAD [31] and MINERv A neutrino [32] and antineutrino [33] CCQE data. Gran et al. [18] applied the model of Nieves et al. to neutrino energies of up to $10 \mathrm{GeV}$. However, conscious of the kinematic limitations of their model, they placed a cut on the three- momentum transfer of $1.2 \mathrm{GeV}$. They compared their results with the MINER $v$ A neutrino and antineutrino CCQE $Q^{2}$ distribution. A similar comparison has been performed also by Mosel et al. [28] using GiBUU. The special implementation of $2 \mathrm{p}-2 \mathrm{~h}$ used in GiBUU is also subject to the same uncertainty at higher energies as in the other approaches. As a general remark, by comparing the results of Refs. [18, 24, 28] with MINERvA data, one can observe that the MINER $v \mathrm{~A} Q^{2}$ distributions can be reproduced also without the inclusion of np-nh excitations. This is not the case of the MiniBooNE $Q^{2}$ distributions $[8,11,24]$. As stressed by Mosel et al., in the case of MINERvA the sensitivity to details of the treatment of np-nh contributions is smaller than the uncertainties introduced by the $Q^{2}$ reconstruction and our insufficient knowledge of pion production. The MINER $v$ A experiment being at higher energies with respect to the MiniBooNE one, the pion production channel becomes in this case more important hence the background subtraction to isolate genuine CCQE and $2 \mathrm{p}-2 \mathrm{~h}$ events is delicate. However by analyzing the energy around the interaction vertex for both neutrino [32] and antineutrino [33] CCQE-like candidates MINER $v$ A collaboration detected additional energy around the vertex in neutrino mode, consistent with an extra proton emission, with respect to the antineutrino mode. This is in agreement with the fact that the strongly correlated initial state nucleon pairs are essentially neutron-proton pairs, leading predominantly to proton-proton (neutron-neutron) pairs in neutrino (antineutrino) charged current reactions, as discussed in Refs. [1, 18, 24, 29]. The GiBUU calculations of Mosel et al. [28] (which include final state interaction for the emitted particles) of the MINER $v$ A neutrino CCQE $Q^{2}$ distribution lead however to different conclusions. Mosel et al. observe that the channels with a pp or a pn pair are very similar, quite flat, and suppressed. An interesting pileup of strength in the Xn channel at small $Q^{2}$ is seen. This is entirely due to the final state interaction of the nucleons.

\section{Pion production and inclusive cross sections}

Turning to the other channels, in this section we rapidly discuss the present situation for the pion production and inclusive cross sections. In the one pion production channel some questions are still open. For instance, as shown in Refs. [34, 35], the MiniBooNE data [36, 37] seem to favor calculations without the inclusion of the pion final state interaction. This doesn't seem to be the case for the MINERvA [38] results. Furthermore there is a general tendency of theoretical calculations and Monte Carlo results to underestimate the MiniBooNE data and to overestimate the MINERvA ones. This is discussed in detail by Sobczyk et al. in Ref. [39] in the case of NuWro generator. Further investigations are needed. We remind the different energies of MiniBooNE and MINERvA neutrino beams. Concerning the coherent pion production channel, K2K and SciBooNE did not observe coherent $\pi^{+}$production at neutrino energies of $\simeq 1 \mathrm{GeV}$. Recently MINER $v$ A [40] and ArgoNeut [41] see evidence for CC coherent pion production at higher energies. In the coherent channel an open puzzle is the ratio of charged current $\pi^{+}$production over the neutral current $\pi^{0}$ production measured by SciBooNE [42] which differs by one order of magnitude with respect to the theoretical predictions [43]. 
The inclusive $v_{\mu}$ CC double differential cross section on carbon has been published by $\mathrm{T} 2 \mathrm{~K}$ in Ref. [7]. The inclusive measurements are important because they are less affected by background subtraction with respect to exclusive channels measurements. Martini and Ericson have compared their predictions with the T2K experimental results in Ref. [12]. In this paper they have shown that in order to obtain an agreement with the T2K inclusive data one needs to consider not only the genuine quasielastic and the one pion production channels but also the multinucleon excitations. These results represent the first successful test of the necessity of the multinucleon emission channel in an experiment with another neutrino flux with respect to the one of MiniBooNE.

\section{Neutrino energy reconstruction problems and neutrino oscillations}

The neutrino energy reconstruction problem has been already mentioned in the introduction. The determination of the initial neutrino energy is commonly done through the charged current neutrino-nucleus quasielastic-like events. For these events where only the charged lepton is observed, the only measurable quantities are then its direction, i.e., its emission angle $\theta$ with respect to the neutrino beam direction and its energy $E_{l}$ (or kinetic energy $T_{l}$ and momentum $P_{l}$ ). The neutrino energy $E_{v}$ is unknown. The usual reconstruction procedure assumes that we are dealing with a genuine quasielastic event on a nucleon at rest. The quasielastic condition then gives the value $\overline{E_{v}}$ of the reconstructed energy: $\overline{E_{v}}=\frac{E_{l}-m_{l}^{2} /(2 M)}{1-\left(E_{l}-P_{l} \cos \theta\right) / M}$. Several nuclear effects can influence this expression. The most important are the np-nh events which have no reason to fulfill the quasielastic relation. This means that for a given set of lepton variables, $E_{l}$ and $\theta$, an infinity of neutrino energy values, instead of the unique quasielastic value implemented in the neutrino energy reconstruction formula, is possible.

Data on neutrino oscillation often involve reconstructed neutrino energies while the analysis implies the real neutrino energy. The corrections corresponding to the transformation from real to reconstructed energy and viceversa are discussed in details in Refs. [9, 10, 16, 27] to which we refer the reader. Here we just summarize some of the main results following the approach of Refs. [9, 10]. Starting from a theoretical distribution expressed with real energies, i.e. the product of the neutrino cross section $\sigma\left(E_{V}\right)$ by the neutrino energy distribution of the beam $\Phi\left(E_{v}\right)$, a smearing procedure to deduce the corresponding distribution of the events, $D_{\text {rec }}\left(\overline{E_{v}}\right)$, in terms of the reconstructed energy can be performed. This distribution can be expressed in terms of the double differential neutrino-nucleus cross section, according to

$$
D_{\text {rec }}\left(\overline{E_{v}}\right)=\int d E_{v} \Phi\left(E_{v}\right) \int_{E_{l}^{\min }}^{E_{l}^{\max }} d E_{l} \frac{M E_{l}-m_{l}^{2} / 2}{\bar{E}_{v}^{2} P_{l}}\left[\frac{d^{2} \sigma}{d \omega d \cos \theta}\right]_{\omega=E_{v}-E_{l}, \cos \theta=\cos \theta\left(E_{l}, \overline{E_{v}}\right)} .
$$

This expression involves the neutrino flux distribution $\Phi\left(E_{V}\right)$, hence the neutrino oscillation parameters. The second integral on the r.h.s. of Eq. (4.1) is denoted as $d\left(E_{v}, \overline{E_{v}}\right)$. It represents the spreading function and depends on $E_{v}$ and $\overline{E_{v}}$. Some examples of its $\overline{E_{v}}$ dependence for several $E_{v}$ values are given in Fig. 3. As one can observe this spreading function is not symmetrical around $E_{v}$. The multinucleon excitations play a crucial role since they create a low energy tail. Similar results have been obtained in Refs. [16, 27].

In Fig. 4 is shown the application of the smearing procedure of Ref. [10] to the two distributions measured in $\mathrm{T} 2 \mathrm{~K}$ : the muonic distributions in the near detector (ND) and far detector 


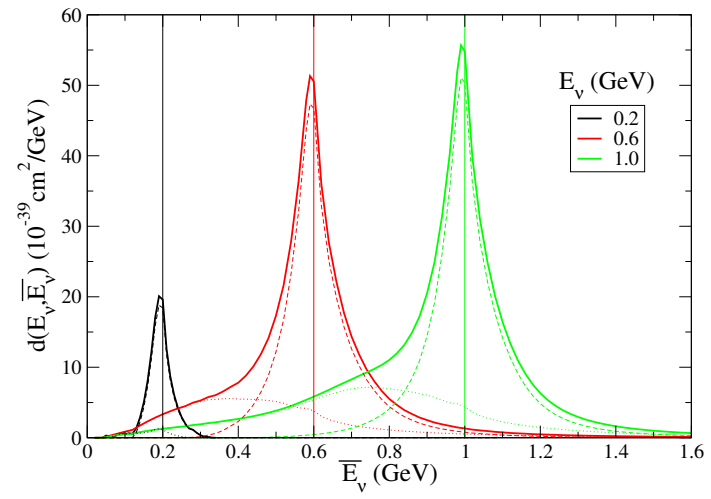

Figure 3: The spreading function per neutron of ${ }^{12} \mathrm{C}$ evaluated for three $E_{v}$ values. The genuine quasielastic (dashed lines) and the multinucleon (dotted lines) contributions are also shown separately. The figure is taken from Ref. [10].

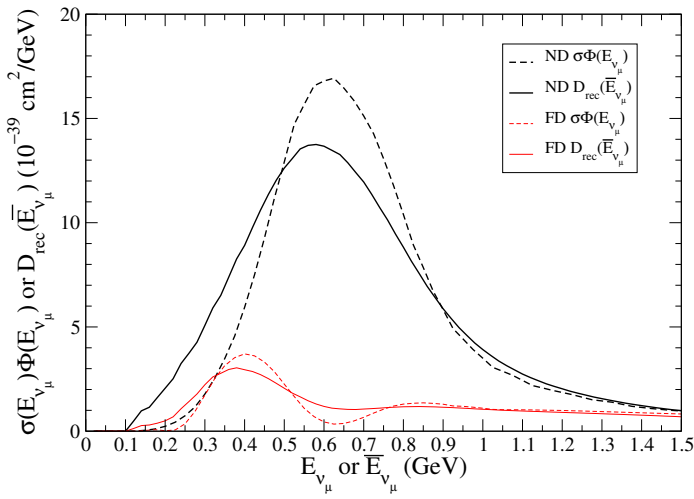

Figure 4: $\mathrm{T} 2 \mathrm{~K}$ distributions per neutrons of muon events before (dashed lines) and after (continuous lines) the energy reconstruction correction in the near and far detector. The figure is taken from Ref. [10].

(FD), hence related to the $v_{\mu}$ disappearance studies. The influence of the reconstructed energy corrections is such that the events tend to escape from the region of high fluxes with a tendency to concentrate at lower energies. Furthermore the middle hole of the far detector results is largely filled. These effects are due to the multinucleon component of the quasielastic-like cross section. Similar results have been obtained in Ref. [27]. The effects are such that an analysis which takes into account the smearing effect is likely to lead to some increase of the oscillation mass value. Recently the T2K collaboration performed an analysis of the $v_{\mu}$ disappearance results by taking into account the multinucleon emission channel [44]. The conclusion of the T2K analysis is that for the present exposure, the multinucleon effect can be ignored, but future analyses will need to incorporate these effects in their model of neutrino-nucleus interactions. This conclusion deserves some comments. First of all, this analysis has been performed by considering the multinucleon contributions as calculated by Nieves et al. which, as it has been shown previously, are smaller with respect to the calculations of Martini et al. Second, part of non-genuine quasielastic events, the ones related to the non pionic decay of the Delta in the nuclear medium, was already included in the Monte Carlo (NEUT) employed for the T2K analysis. Further studies are needed. Work in this direction is in progress.

\section{References}

[1] M. Martini, M. Ericson, G. Chanfray and J. Marteau, Phys. Rev. C 80, 065501 (2009).

[2] M. Martini, M. Ericson, G. Chanfray and J. Marteau, Phys. Rev. C 81, 045502 (2010).

[3] A. A. Aguilar-Arevalo et al. [MiniBooNE Collaboration], Phys. Rev. D 81, 092005 (2010).

[4] J. Marteau, J. Delorme and M. Ericson, Nucl. Instrum. Meth. A 451, 76 (2000).

[5] A. A. Aguilar-Arevalo et al. [MiniBooNE Collaboration], Phys. Rev. D 88, no. 3, 032001 (2013).

[6] Y. Nakajima et al. [SciBooNE Collaboration], Phys. Rev. D 83, 012005 (2011).

[7] K. Abe et al. [T2K Collaboration], Phys. Rev. D 87, 092003 (2013). 
[8] M. Martini, M. Ericson and G. Chanfray, Phys. Rev. C 84, 055502 (2011).

[9] M. Martini, M. Ericson and G. Chanfray, Phys. Rev. D 85, 093012 (2012).

[10] M. Martini, M. Ericson and G. Chanfray, Phys. Rev. D 87, 013009 (2013).

[11] M. Martini and M. Ericson, Phys. Rev. C 87, 065501 (2013).

[12] M. Martini and M. Ericson, Phys. Rev. C 90, 025501 (2014).

[13] M. Ericson and M. Martini, Phys. Rev. C 91, no. 3, 035501 (2015).

[14] J. Nieves, I. Ruiz Simo, M. J. Vicente Vacas, Phys. Rev. C83, 045501 (2011).

[15] J. Nieves, I. Ruiz Simo and M. J. Vicente Vacas, Phys. Lett. B 707, 72 (2012).

[16] J. Nieves, F. Sanchez, I. Ruiz Simo and M. J. Vicente Vacas, Phys. Rev. D 85, 113008 (2012).

[17] J. Nieves, I. Ruiz Simo and M. J. Vicente Vacas, Phys. Lett. B 721, 90 (2013).

[18] R. Gran, J. Nieves, F. Sanchez and M. J. Vicente Vacas, Phys. Rev. D 88, 113007 (2013).

[19] J. E. Amaro, M. B. Barbaro, J. A. Caballero, T. W. Donnelly et al. Phys. Lett. B696, 151-155 (2011).

[20] J. E. Amaro, M. B. Barbaro, J. A. Caballero, T. W. Donnelly et al. Phys. Rev. D 84, 033004 (2011).

[21] J. E. Amaro, M. B. Barbaro, J. A. Caballero and T. W. Donnelly, Phys. Rev. Lett. 108, 152501 (2012).

[22] I. Ruiz Simo, C. Albertus, J. E. Amaro, M. B. Barbaro et al. Phys. Rev. D 90, no. 3, 033012 (2014).

[23] I. R. Simo, C. Albertus, J. E. Amaro, M. B. Barbaro et al. Phys. Rev. D 90, no. 5, 053010 (2014).

[24] G. D. Megias, T. W. Donnelly, O. Moreno et al. Phys. Rev. D 91, no. 7, 073004 (2015).

[25] A. Bodek, H. S. Budd and M. E. Christy, Eur. Phys. J. C 71, 1726 (2011).

[26] O. Lalakulich, K. Gallmeister and U. Mosel, Phys. Rev. C 86, no. 1, 014614 (2012).

[27] O. Lalakulich, U. Mosel and K. Gallmeister, Phys. Rev. C 86, 054606 (2012).

[28] U. Mosel, O. Lalakulich and K. Gallmeister, Phys. Rev. D 89, no. 9, 093003 (2014).

[29] A. Lovato, S. Gandolfi, J. Carlson, S. C. Pieper, R. Schiavilla, Phys. Rev. Lett. 112, 182502 (2014).

[30] A. Lovato, S. Gandolfi, J. Carlson, S. C. Pieper and R. Schiavilla, arXiv:1501.01981 [nucl-th].

[31] V. Lyubushkin et al. [NOMAD Collaboration], Eur. Phys. J. C 63, 355 (2009).

[32] G. A. Fiorentini et al. [MINERvA Collaboration], Phys. Rev. Lett. 111, 022502 (2013).

[33] L. Fields et al. [MINERvA Collaboration], Phys. Rev. Lett. 111, no. 2, 022501 (2013).

[34] O. Lalakulich and U. Mosel, Phys. Rev. C 87, 014602 (2013).

[35] E. Hernandez, J. Nieves and M. J. V. Vacas, Phys. Rev. D 87, 113009 (2013).

[36] A. A. Aguilar-Arevalo et al. [MiniBooNE Collaboration], Phys. Rev. D 81, 013005 (2010).

[37] A. A. Aguilar-Arevalo et al. [MiniBooNE Collaboration], Phys. Rev. D 83, 052007 (2011).

[38] B. Eberly et al. [MINERvA Collaboration], arXiv:1406.6415 [hep-ex].

[39] J. T. Sobczyk and J. Zmuda, Phys. Rev. C 91, 045501 (2015).

[40] A. Higuera et al. [MINERvA Collaboration], Phys. Rev. Lett. 113, 261802 (2014).

[41] R. Acciarri et al. [ArgoNeuT Collaboration], Phys. Rev. Lett. 113, 261801 (2014).

[42] Y. Kurimoto et al. [SciBooNE Collaboration], Phys. Rev. D 81, 111102 (2010).

[43] L. Alvarez-Ruso, Y. Hayato and J. Nieves, New J. Phys. 16, 075015 (2014).

[44] K. Abe et al. [T2K Collaboration], Phys. Rev. D 91, 072010 (2015). 